\title{
Cryptococcal meningoencephalitis in patients with mantle cell lymphoma on ibrutinib
}

\author{
Kai Sun ${ }^{1}$, Saro Kasparian ${ }^{1}$, Swaminathan Iyer $^{2}$ and Sai Ravi Pingali ${ }^{2}$ \\ 'Department of Medicine, Houston Methodist Hospital, Houston, TX 77030, USA \\ 2Department of Hematology, Houston Methodist Hospital, Houston, TX 77030, USA \\ Correspondence to: Sai Ravi Pingali. Email: spingali@houstonmethodist.org
}

\begin{abstract}
Ibrutinib, a Bruton's tyrosine kinase inhibitor, has been increasingly widely used in relapsed and refractory mantle cell lymphoma (MCL) and chronic lymphocytic leukaemia [1,2]. With its use becoming more common, there have been emerging case reports of opportunistic infections like cryptococcal infections [3-8]. These infections in patients receiving ibrutinib were mostly reported in patients with chronic lymphocytic leukaemia, who have poor immune reconstitution. Here, we report two cases of cryptococcal meningoencephalitis in patients with MCL on ibrutinib.
\end{abstract}

Keywords: cryptococcal meningoencephalitis, mental cell lymphoma, ibrutinib

ecancer 2018, 12:836 https://doi.org/10.3332/ecancer.2018.836

Copyright: (c) the authors; licensee ecancermedicalscience. This is an Open Access article distributed under the terms of the Creative Commons Attribution License (http://creativecommons.org/licenses/by/3.0), which permits unrestricted use, distribution, and reproduction in any medium, provided the original work is properly cited. 


\section{Case 1}

A 70-year-old male with a past medical history of type II diabetes and primary refractory stage IV B mantle cell lymphoma (MCL) presented with altered mental status for 2 weeks. The patient was treated with three cycles of R-CHOP (rituximab, cyclophosphamide, doxorubicin, vincristine and prednisone) without response. Following that, he was treated with bortezomib and rituximab with partial response. Five months prior to presentation, the chemotherapy regimen was changed to ibrutinib 560 mg daily with rituximab 375 mg weekly for 4 weeks followed by rituximab monthly. Five months after he had been on ibrutinib and rituximab, he presented with a 2-week history of altered mental status, visual hallucination that was worse at night and headache. He denied fevers, chills, nausea, vomiting or diarrhoea. Laboratory results revealed sodium of $126 \mathrm{mEq} / \mathrm{L}$, normal white blood cell (WBC) of $5.15 \times 10^{9} / \mathrm{L}$ with $81 \%$ neutrophils and $12 \%$ lymphocytes, normocytic normochromic anaemia with red blood cell (RBC) of 2.79 , haemoglobin of $9.2 \mathrm{~g} / \mathrm{dL}$ and thrombocytopenia with platelet of $95 \times 10^{9} / \mathrm{L}$. Magnetic resonance imaging (MRI) of the brain with and without contrast revealed two punctate foci of enhancement in the posterior left basal ganglia measuring 2-3 mm and $1.5 \mathrm{~mm}$, respectively. His altered mental status was initially attributed to hyponatraemia, and the patient was started on a fluid restricted diet, sodium tablet and later tolvaptan. However, his symptoms, including headache and visual hallucinations, didn't improve and the patient started having low grade fevers. Lumbar puncture was done 1 week after hospitalisation which showed slightly hazy pale yellow cerebral spinal fluids (CSFs), decreased glucose of $36 \mathrm{mg} / \mathrm{dL}$, elevated protein of 159 $\mathrm{mg} / \mathrm{dL}, 571 \mathrm{WBC}$ with $41 \%$ lymphocytes and 59\% neutrophils, 1107 RBC. CSF cryptococcal antigen titre was 1:2 and serum cryptococcal antigen titre was 1:64. No growth on CSF fungal culture. Ibrutinib was discontinued. The patient was started on amphotericin 3 mg/kg/ day and flucytosine 1500 mg every 8 hours for 14 days with improvement of symptoms and transitioned to oral fluconazole 400 mg twice a day. Repeated serum cryptococcal antigen titres on follow-up visits kept trending down till nondetectable. He was continued on fluconazole and was started on lenalidomide and rituximab later for the MCL.

\section{Case 2}

A 78-year-old male with a past medical history of stage IV MCL presented with imbalance, headache behind the eyes and double vision that progressively got worse within a period of several months. The patient developed fevers later after admission. His stage IV MCL was diagnosed in 2006 with bone marrow involvement and was heavily treated with multiple chemotherapies, including R-CHOP, bortezomib and rituximab, bendamustine and rituximab and Bexxar and lenalidomide from 2006 to 2013. Ibrutinib 540 mg daily was initiated after his third relapse 2 years prior to his presentation and he had remained in complete remission. Laboratory findings revealed leukopenia with WBC of $1.51 \times 10^{9} / \mathrm{L}$ with $68.9 \%$ neutrophils and $17.7 \%$ lymphocytes, anaemia with haemoglobin of $9.5 \mathrm{~g} / \mathrm{dL}$, thrombocytopenia with platelet of $89 \times 10^{9} / \mathrm{L}$. MRI brain with and without contrast showed vasogenic oedema in the right parieto-occipital region with overlying gyral thickening and gyriform enhancement with mild regional mass effect. Patient subsequently underwent lumbar puncture with opening pressure of $21-\mathrm{cm}$ water. CSF was clear with normal glucose of $42 \mathrm{mg} / \mathrm{dL}$, elevated protein of $61 \mathrm{mg} / \mathrm{dL}, 18 \mathrm{WBC}$ with $78 \%$ lymphocytes, $19 \%$ monocytes and $3 \%$ neutrophils and 12 RBC. CSF cryptococcal antigen was positive while fungal smear and cultures showed no growth. Ibrutinib was discontinued. He was started on amphotericin and flucytosine for 14 days with an improvement of his symptoms and was continued on oral fluconazole. Repeated lumbar puncture 2 weeks after the initiation of treatment showed normal glucose of $49 \mathrm{mg} / \mathrm{dL}$, normal protein of $41 \mathrm{mg} / \mathrm{dL}, 4 \mathrm{WBC}$ and $1 \mathrm{RBC}$. The CSF cryptococcal antigen became negative. The patient remained in remission off ibrutinib or other medications for MCL until 4 months later when he was found to have therapy-related myelodysplastic syndrome which later transformed to acute myeloid leukaemia.

\section{Discussion}

Cryptococcal infection is an opportunistic fungal infection with a predilection to the lungs and central nervous system [9, 10]. The majority of cases of cryptococcal infection were Cryptococcal neoformans and most often happened in patients with human immunodeficiency virus (HIV) infection. The cases that occurred in non-HIV-infected individuals were transplant recipients, patients who are receiving 
immunosuppressive agents such as glucocorticosteroids and cytotoxic chemotherapy and patients with innate immunologic problems [11]. It was found that cell immunity plays an essential role in preventing and controlling the cryptococcal infections [9, 10].

Bruton's tyrosine kinase (BTK) is a cytoplastic tyrosine kinase of the Tec family that is essential for B-cell receptor signalling and B-cell survival [12]. Ibrutinib works as an orally active inhibitor of BTK that covalently binds to cysteine Cys-481 of BTK and irreversibly inactivates the kinase [12, 13]. Ibrutinib has shown its effect in treating chronic lymphocytic leukaemia (CLL), MCL and other B-cell lymphoproliferative diseases [1, 2]. However, emerging cases of cryptococcal infections in patients on ibrutinib have been reported, most of which were patients with CLL.

Okamoto et al [8] reported a case of disseminated cryptococcosis in a patient with CLL after being on ibrutinib for 1 month. Messina et al [7] later reported two cases of cryptococcal infection involving central nervous system in a patient with indolent lymphoma and one patient with CLL. Both patients presented shortly after they were started on ibrutinib (3 weeks and 1 month, respectively). Another retrospective study done by Varughese et al [14] which looked at serious infections in the patients receiving ibrutinib found that 16/378 developed opportunistic fungal infections. Ibrutinib was started within 1 year in most of the patients. Ajam et al [3] reported a primary cutaneous case in a patient with relapsed CLL. A large retrospective study performed by Rogers et al [15] which looked at the opportunistic infections in the patients on ibrutinib revealed that one CLL patient out of 566 patients with haematologic malignancies had cryptococcal infection.

Cases of cryptococcal infection in the MCL patients on ibrutinib have been scarcely reported. It was observed that ibrutinib inhibits antigen receptor signalling in B cells not T cells [16]. As mentioned above, cell immunity plays an important role in cryptococcal infection and should be relatively intact in the MCL patients who are on cytotoxic chemotherapies. One mechanism of the infection might be ibrutinib's indirect effect on cell immunity. BTK has been demonstrated to participate in the regulation of nitric oxide induction and bactericidal functions in macrophages [17] which interact with helper cells and participate in cell immunity. Ibrutinib might indirectly affect cell immunity by inhibiting cells that interact with T cell. Szymczak et al [18] found that X-linked immunodeficiency mice were not able to contain cryptococcus infection in the lungs due to reduced uptake by macrophages. The inhibition of macrophages by ibrutinib could be another mechanism.

\section{Conclusions}

The majority of previous case reports about cryptococcal infections in patients with haematologic malignancies on ibrutinib were patients with CLL. In addition, the onset of symptoms was shortly after they were started on ibrutinib. Both of our patients had MCL which was not known to be associated with cell immunity deficiency, and cryptococcal infections were scarcely reported in MCL patients on ibrutinib. They had been on ibrutinib for 5 months and 2 years, respectively, before they presented with symptoms, which also distinguishes our report from previous case reports. As ibrutinib gains more popularity in $\mathrm{MCL}$, it is important for physicians to stay vigilant to keep cryptococcal infection in the differentials even after patients have been on this medication for a relatively long period of time so that timely diagnosis and treatment can be implemented.

\section{Conflicts of interest}

The authors declare that they have no conflicts of interest.

\section{Funding acknowledgment}

This research received no specific grant from any funding agency in the public, commercial or not-for-profit sectors. 


\section{References}

1. Burger JA, Tedeschi A, and Barr PM, et al (2015) Ibrutinib as initial therapy for patients with chronic lymphocytic leukemia N Engl J Med 373(25) 2425-2437

2. Wang ML, Rule S, and Martin P, et al (2013) Targeting BTK with ibrutinib in relapsed or refractory mantle-cell lymphoma N Engl J Med 369(6) 507-516 https://doi.org/10.1056/NEJMoa1306220 PMID: 23782157 PMCID: 4513941

3. Ajam T, Hyun G, and Blue B, et al (2016) Primary cutaneous cryptococcosis in a patient with chronic lymphocytic leukemia: a case report Ann Hematol Oncol 3(3) 1082

4. Baron M, Zini JM, and Challan Belval T, et al (2017) Fungal infections in patients treated with ibrutinib: two unusual cases of invasive aspergillosis and cryptococcal meningoencephalitis Leuk Lymphoma 58(12) 2981-2982 https://doi.org/10.1080/10428 194.2017.1320710 PMID: $\underline{28554246}$

5. Devirgiliis V, Panasiti V, and Borroni RG, et al (2008) Cutaneous cryptococcosis in a patient affected by chronic lymphocytic leukaemia: a case report Int J Immunopathol Pharmacol 21(2) 463-466 https://doi.org/10.1177/039463200802100227 PMID: 18547493

6. Dizdar OS, Karakecili F, and Coskun BN, et al (2012) Fatal cryptococcal meningitis in a patient with chronic lymphocytic leukemia Mediterr J Hematol Infect Dis 4(1) e2012039 https://doi.org/10.4084/mjhid.2012.039 PMID: 22811788 PMCID: 3395708

7. Messina JA, Maziarz EK, and Spec A, et al (2017) Disseminated cryptococcosis with brain involvement in patients with chronic lymphoid malignancies on ibrutinib Open Forum Infect Dis 4(1) ofw261 PMID: 28480254 PMCID: $\underline{5413986}$

8. Okamoto K, Proia LA, and Demarais PL (2016) Disseminated cryptococcal disease in a patient with chronic lymphocytic leukemia on ibrutinib Case Rep Infect Dis 20164642831 PMID: 27703818 PMCID: 5039271

9. Chayakulkeeree M and Perfect JR (2006) Cryptococcosis Infect Dis Clin North Am 20(3) 507-544, v-vi https://doi.org/10.1016/j. idc.2006.07.001 PMID: 16984867

10. Li SS and Mody CH (2010) Cryptococcus Proc Am Thorac Soc 7(3) 186-196 https://doi.org/10.1513/pats.200907-063AL PMID: 20463247

11. Pappas PG (2013) Cryptococcal infections in non-HIV-infected patients Trans Am Clin Climatol Assoc 124 61-79 PMID: 23874010 PMCID: $\underline{3715903}$

12. Buggy JJ and Elias L (2012) Bruton tyrosine kinase (BTK) and its role in B-cell malignancy Int Rev Immuno/ 31(2) 119-132 https:// doi.org/10.3109/08830185.2012.664797 PMID: 22449073

13. Wiestner A (2013) Targeting B-Cell receptor signaling for anticancer therapy: the Bruton's tyrosine kinase inhibitor ibrutinib induces impressive responses in B-cell malignancies J Clin Oncol 31(1) 128-130 https://doi.org/10.1200/JCO.2012.44.4281

14. Varughese T, Taur $Y$, and Cohen N, et al (2018) Serious infections in patients receiving ibrutinib for treatment of lymphoid malignancies Clin Infect Dis https://doi.org/10.1093/cid/ciy175 PMID: 29509845

15. Rogers KA, Luay M, Zhao Q, et al (2017) Incidence and type of opportunistic infections during ibrutinib treatment at a single academic center Blood 130830

16. Honigberg LA, Smith AM, and Sirisawad M, et al (2010) The Bruton tyrosine kinase inhibitor PCI-32765 blocks B-cell activation and is efficacious in models of autoimmune disease and B-cell malignancy Proc Natl Acad Sci U S A 107(29) 13075-13080 https://doi.org/10.1073/pnas.1004594107 PMID: 20615965 PMCID: 2919935

17. Mukhopadhyay S, Mohanty M, and Mangla A, et al (2002) Macrophage effector functions controlled by Bruton's tyrosine kinase are more crucial than the cytokine balance of T cell responses for microfilarial clearance J Immunol 168(6) 2914-2921 https:// doi.org/10.4049/jimmunol.168.6.2914 PMID: 11884462

18. Szymczak WA, Davis MJ, and Lundy SK, et al (2013) X-linked immunodeficient mice exhibit enhanced susceptibility to Cryptococcus neoformans infection MBio 4(4) https://doi.org/10.1128/mBio.00265-13 PMID: 23820392 PMCID: $\underline{3705448}$ 